\author{
NOMENCLATURE UPDATE
}

\title{
Nomenclature for factors of the HLA system, update December 2009
}

\author{
Compiled by Steven G. E. Marsh for the WHO Nomenclature Committee for Factors of the HLA System. \\ Correspondence: Steven G. E. Marsh, Anthony Nolan Research Institute, Royal Free Hospital, Pond Street, London NW3 20G, United Kingdom. \\ Tel: +44 207284 8321; Fax: +44 207284 8331; e-mail: steven.marsh@ucl.ac.uk; URLs: www. hla.alleles.org, www.anthonynolan.org.uk/HIG, \\ www.ebi.ac.uk/imgt/hla \\ doi: 10.1111/j.1399-0039.2010.01462.x
}

The following sequences have been submitted to the Nomenclature Committee since the November 2009 nomenclature update and, following agreed policy, have been assigned official allele designations. Full details of all sequences will be published in a forthcoming report.
Below are listed the newly assigned sequences (Table 1) and confirmations of previously reported sequences (Table 2). The accession number of each sequence is given and these can be used to retrieve the sequence files from the EMBL, GenBank, Vega or DDBJ data libraries. Although accession numbers

Table 1 New Sequences

\begin{tabular}{|c|c|c|c|}
\hline Sequence & Cell identification & Accession number & Submitting author \\
\hline$A * 0372$ & JMDP01K043 & AB535153 & Kenji Tadokoro, Tokyo, Japan \\
\hline$A^{*} 0373$ & BY00578 & GU256011 & Carolyn K Hurley, Washington DC, USA \\
\hline$A^{*} 0374$ & BY00575 & GU256014 & Carolyn K Hurley, Washington DC, USA \\
\hline$A^{*} 2511$ & BY00577 & GU256012 & Carolyn K Hurley, Washington DC, USA \\
\hline$A * 300205$ & BY00580 & GU256009 & Carolyn K Hurley, Washington DC, USA \\
\hline$A * 3033$ & JMDP01K045 & AB535154 & Kenji Tadokoro, Tokyo, Japan \\
\hline$A * 3034$ & BY00564 & GU138062 & Carolyn K Hurley, Washington DC, USA \\
\hline$A * 3221$ & BY00581 & GU256008 & Carolyn K Hurley, Washington DC, USA \\
\hline$A * 3605$ & 170705 & FN557302 & Elizabeth Keller, Munich, Germany \\
\hline$A * 927102$ & BY00576 & GU256013 & Carolyn K Hurley, Washington DC, USA \\
\hline$B * 1336$ & JMDP36K066 & AB536746 & Kenji Tadokoro, Tokyo, Japan \\
\hline$B * 400209$ & JMDP36K061 & AB536744 & Kenji Tadokoro, Tokyo, Japan \\
\hline$B * 5189$ & JMDP36K062 & AB536745 & Kenji Tadokoro, Tokyo, Japan \\
\hline$B * 5828$ & BY00573 & GU256006 & Carolyn K Hurley, Washington DC, USA \\
\hline$B * 9586$ & JMDP36K033 & AB477102 & Kenji Tadokoro, Tokyo, Japan \\
\hline $\mathrm{CW}^{*} 0629$ & OUAL & FN597419 & Valerie Dubois, Lyon, France \\
\hline$C W^{*} 1526$ & SZBM685 & GU232859 & Zhihui Deng, ShenZhen, China \\
\hline DRB1 ${ }^{*} 040104$ & HN-88028-8 & FJ438928 & Histogenetics, Ossining, USA \\
\hline DRB1*040105 & HN-20315-2 & FJ438930 & Histogenetics, Ossining, USA \\
\hline DRB1*040106 & HN-57737-8 & FJ549416 & Histogenetics, Ossining, USA \\
\hline DRB1*040305 & HN-09532-5 & FJ438927 & Histogenetics, Ossining, USA \\
\hline DRB $1 * 040403$ & HN-37506-5, HN-78268-8 & FJ438924, FJ594733 & Histogenetics, Ossining, USA \\
\hline DRB1 $1 * 040404$ & $\mathrm{HN}-00728-6$ & FJ438929 & Histogenetics, Ossining, USA \\
\hline DRB1*040509 & HN-8629540 & FJ766004 & Histogenetics, Ossining, USA \\
\hline DRB1*040704 & HN-18682-5, HN-763322 & FJ438923, FJ489881 & Histogenetics, Ossining, USA \\
\hline DRB1*0489 & HN-6790922 & FJ858914 & Histogenetics, Ossining, USA \\
\hline DRB1*115802 & HN-31092-9 & FJ766005 & Histogenetics, Ossining, USA \\
\hline DRB1*130304 & $\mathrm{HN}-64842-5$ & FJ858909, FJ858910 & Histogenetics, Ossining, USA \\
\hline DRB1*132102 & $\begin{array}{l}\text { HN-50459-4, HN-64798-8, HN-65133-7, } \\
\text { HN-55271-7, HN-50682-0, HN-57306-9 }\end{array}$ & $\begin{array}{l}\text { FJ766012, FJ858912, FJ875605, } \\
\text { FJ875606, FJ875607, FJ858911 }\end{array}$ & Histogenetics, Ossining, USA \\
\hline DRB1*1395 & HN-67038-8 & FJ640587 & Histogenetics, Ossining, USA \\
\hline DRB1*1396 & $\mathrm{HN}-66141-1$ & FJ640586 & Histogenetics, Ossining, USA \\
\hline
\end{tabular}


Table 1 Continued

\begin{tabular}{llll}
\hline Sequence & Cell identification & Accession number & Submitting author \\
\hline DRB1*1397 & HN-26392-8 & FJ766011 & Histogenetics, Ossining, USA \\
DRB1*150110 & HN-30368-4 & FJ358155 & Histogenetics, Ossining, USA \\
DRB1*150111 & HN-55222-3, HN-62717-0 & FJ358157, FJ875603 & Histogenetics, Ossining, USA \\
DRB1*150112 & HN-17926-7 & FJ358169 & Histogenetics, Ossining, USA \\
DRB1*1542 & HN-39373-2 & FJ766007 & Histogenetics, Ossining, USA \\
DRB1*1543 & HN-44736290 & FJ858913 & Histogenetics, Ossining, USA \\
\hline
\end{tabular}

Table 2 Confirmatory Sequences

\begin{tabular}{|c|c|c|c|}
\hline Sequence & Cell identification & Accession number & Submitting author \\
\hline$A^{*} 030104$ & JMDP36K031 & AB536869 & Kenji Tadokoro, Tokyo, Japan \\
\hline$A^{*} 0357$ & P-661594 & FN430729 & Rainer Blasczyk, Hannover, Germany \\
\hline$A^{*} 2405$ & JMDP01K069 & AB536870 & Kenji Tadokoro, Tokyo, Japan \\
\hline$A * 2426$ & BY00562 & GU138064 & Carolyn K Hurley, Washington DC, USA \\
\hline A*6806 & BY00563 & GU138063 & Carolyn K Hurley, Washington DC, USA \\
\hline$A^{*} 6807$ & BY00567 & GU144508 & Carolyn K Hurley, Washington DC, USA \\
\hline$A^{*} 680802$ & BY00579 & GU256010 & Carolyn K Hurley, Washington DC, USA \\
\hline$A * 9282$ & AKB-650304 & FN430730 & Rainer Blasczyk, Hannover, Germany \\
\hline$B * 070208$ & HN-46788-1, HN-12328-3 & GQ449646, GQ859541 & Histogenetics, Ossining, USA \\
\hline$B * 070210$ & HN-97825-6 & G0994063 & Histogenetics, Ossining, USA \\
\hline$B^{*} 0777$ & HN-57433-7 & GQ401200 & Histogenetics, Ossining, USA \\
\hline$B * 0780$ & $\mathrm{HN}-24661-7$ & GQ914797 & Histogenetics, Ossining, USA \\
\hline$B * 0787$ & $\begin{array}{l}\text { HN-30108-0, HN-84250-6, HN-30107-2, } \\
\text { HN-20795-6 }\end{array}$ & $\begin{array}{l}\text { GQ245737, GQ254338, GQ345063, } \\
\text { G0994071 }\end{array}$ & Histogenetics, Ossining, USA \\
\hline$B * 080107$ & HN-85124-4 & GQ254351 & Histogenetics, Ossining, USA \\
\hline$B * 080108$ & HN-82787-0, HN-12663-3, HN-60847-6 & GQ240393, GQ449633, G0994057 & Histogenetics, Ossining, USA \\
\hline$B * 080108$ & P-664367 & FN430731 & Rainer Blasczyk, Hannover, Germany \\
\hline$B * 081203$ & HN-98041-3 & GQ240392 & Histogenetics, Ossining, USA \\
\hline$B * 0841$ & HN-98338-7, HN-97818-9 & GQ199710, GQ254343 & Histogenetics, Ossining, USA \\
\hline$B * 1418$ & $\mathrm{HN}-03501-0$ & GQ245747 & Histogenetics, Ossining, USA \\
\hline$B * 150303$ & HN-89393-3, HN-30081-3, HN-89387-5 & GQ149275, GQ199709, GQ245731 & Histogenetics, Ossining, USA \\
\hline$B * 150502$ & HN-92802-4 & GQ245748 & Histogenetics, Ossining, USA \\
\hline$B * 1506$ & BY00560 & GU138066 & Carolyn K Hurley, Washington DC, USA \\
\hline$B * 151002$ & HN-45679-7 & GQ240387 & Histogenetics, Ossining, USA \\
\hline$B * 1535$ & JMDP01K067 & AB536871 & Kenji Tadokoro, Tokyo, Japan \\
\hline$B * 1539$ & JMDP01K068 & AB536872 & Kenji Tadokoro, Tokyo, Japan \\
\hline$B * 1561$ & BY00561 & GU138065 & Carolyn K Hurley, Washington DC, USA \\
\hline$B * 1806$ & BY00559 & GU138067 & Carolyn K Hurley, Washington DC, USA \\
\hline$B * 180702$ & HN-39607-9 & GQ994065 & Histogenetics, Ossining, USA \\
\hline$B * 1833$ & HN-02964-7 & GQ859551 & Histogenetics, Ossining, USA \\
\hline$B * 1835$ & HN-N96546, HN-N260491 & GQ240386, GQ900552 & Histogenetics, Ossining, USA \\
\hline$B * 1847$ & HN-33661-9 & GQ468246 & Histogenetics, Ossining, USA \\
\hline$B * 2720$ & BY00555 & GU138071 & Carolyn K Hurley, Washington DC, USA \\
\hline$B * 2751$ & HN-62875-5 & GQ900555 & Histogenetics, Ossining, USA \\
\hline$B * 2760$ & HN-90373-2 & GQ149277 & Histogenetics, Ossining, USA \\
\hline$B * 350111$ & HN-21479-7, HN-39298-0 & GQ345060, GQ401196 & Histogenetics, Ossining, USA \\
\hline$B * 350119$ & HN-83813-6 & GQ900547 & Histogenetics, Ossining, USA \\
\hline$B * 350804$ & HN-25909-1 & GQ449635 & Histogenetics, Ossining, USA \\
\hline$B * 3511$ & BY00556 & GU138070 & Carolyn K Hurley, Washington DC, USA \\
\hline$B * 3521$ & JMDP36K043 & AB536873 & Kenji Tadokoro, Tokyo, Japan \\
\hline$B * 3564$ & JMDP36K045 & AB536874 & Kenji Tadokoro, Tokyo, Japan \\
\hline$B * 3598$ & HN-61859-0 & GQ468249 & Histogenetics, Ossining, USA \\
\hline$B * 3717$ & $\mathrm{HN}-04271-5$ & GQ859545 & Histogenetics, Ossining, USA \\
\hline$B * 390110$ & HN-31356-8 & GQ245750 & Histogenetics, Ossining, USA \\
\hline$B * 3912$ & BY00558 & GU138068 & Carolyn K Hurley, Washington DC, USA \\
\hline$B * 3914$ & BY00557 & GU138069 & Carolyn K Hurley, Washington DC, USA \\
\hline
\end{tabular}


Table 2 Continued

\begin{tabular}{|c|c|c|c|}
\hline Sequence & Cell identification & Accession number & Submitting author \\
\hline$B * 3915$ & BY00568 & GU144507 & Carolyn K Hurley, Washington DC, USA \\
\hline$B * 400207$ & HN-N260568 & GQ859532 & Histogenetics, Ossining, USA \\
\hline$B * 4011$ & JMDP01K063 & AB537164 & Kenji Tadokoro, Tokyo, Japan \\
\hline$B * 4026$ & BY00569 & GU256002 & Carolyn K Hurley, Washington DC, USA \\
\hline$B * 4029$ & JMDP36K042 & AB537165 & Kenji Tadokoro, Tokyo, Japan \\
\hline$B * 4210$ & HN-21017-8 & GQ468252 & Histogenetics, Ossining, USA \\
\hline$B * 440206$ & HN-N261934 & GQ491085 & Histogenetics, Ossining, USA \\
\hline$B * 440209$ & HN-96331-0 & GQ245739 & Histogenetics, Ossining, USA \\
\hline$B * 440210$ & HN-34788-2, HN-50429-6 & GQ859550, GU017931 & Histogenetics, Ossining, USA \\
\hline$B * 4418$ & BY00554 & GU138072 & Carolyn K Hurley, Washington DC, USA \\
\hline$B * 4474$ & HN-67794-6 & GQ254352 & Histogenetics, Ossining, USA \\
\hline$B * 4486$ & $\mathrm{HN}-41994-1$ & GQ254335 & Histogenetics, Ossining, USA \\
\hline$B * 4496$ & HN-33582-4 & GO254349 & Histogenetics, Ossining, USA \\
\hline$B * 510110$ & HN-73186-2 & GQ401191 & Histogenetics, Ossining, USA \\
\hline$B * 5165$ & $\begin{array}{l}\text { HN-N94065, HN-25984-8, HN-N262510, } \\
\text { HN-N035688, HN-N261989, } \\
\text { HN-N262508 }\end{array}$ & $\begin{array}{l}\text { GQ245728, GQ449634, GQ859535, } \\
\text { GQ914788, GQ914790, G0914791 }\end{array}$ & Histogenetics, Ossining, USA \\
\hline$B * 5167$ & $\mathrm{HN}-75991-0$ & GQ245733 & Histogenetics, Ossining, USA \\
\hline$B * 5168$ & HN-62842-2 & G0914793 & Histogenetics, Ossining, USA \\
\hline$B * 5169$ & HN-77817-6 & GQ240391 & Histogenetics, Ossining, USA \\
\hline$B * 5175$ & $\begin{array}{l}\text { HN-N257741, HN-N263825, } \\
\text { HN-N034218 }\end{array}$ & GQ468247, GQ859531, G0994058 & Histogenetics, Ossining, USA \\
\hline$B * 5182$ & $\mathrm{HN}-17946-0, \mathrm{HN}-17947-8$ & GQ449643, GQ449644 & Histogenetics, Ossining, USA \\
\hline$B * 550105$ & HN-53197-2 & Go914792 & Histogenetics, Ossining, USA \\
\hline$B * 550106$ & $\mathrm{HN}-42409-1$ & GQ491084 & Histogenetics, Ossining, USA \\
\hline$B * 560102$ & HN-87472-3 & G0994062 & Histogenetics, Ossining, USA \\
\hline$B * 5602$ & BY00553 & GU138073 & Carolyn K Hurley, Washington DC, USA \\
\hline$B * 570105$ & $H N-59275-0$ & GQ859549 & Histogenetics, Ossining, USA \\
\hline$B * 5726$ & HN-94214-4 & GQ859540 & Histogenetics, Ossining, USA \\
\hline$B * 5904$ & JMDP01K054 & AB537166 & Kenji Tadokoro, Tokyo, Japan \\
\hline$B * 9553$ & $\begin{array}{l}\text { HN-88641-3, HN-94111-9, HN-86738-9, } \\
\text { HN-35520-8 }\end{array}$ & $\begin{array}{l}\text { GQ149278, GQ245745, GQ254341, } \\
\text { G0859547 }\end{array}$ & Histogenetics, Ossining, USA \\
\hline$B * 9581 \mathrm{~N}$ & HN-95028-4 & GQ245715 & Histogenetics, Ossining, USA \\
\hline $\mathrm{CW}^{*} 010207$ & HN-81703-5, HN-27905-9, HN-28170-6 & GQ180267, GQ180445, GQ254397 & Histogenetics, Ossining, USA \\
\hline $\mathrm{CW}^{*} 010208$ & $\begin{array}{l}\text { HN-02906-7, HN-55932-2, HN-53240-0, } \\
\text { HN-35686-3, HN-57785-8, } \\
\text { HN-99074-6, HN-87701-9 }\end{array}$ & $\begin{array}{l}\text { GU128019, GQ180241, GQ180248, } \\
\text { GQ180389, GQ180411, GQ240487, } \\
\text { GQ254390 }\end{array}$ & Histogenetics, Ossining, USA \\
\hline$C w^{*} 0110$ & BY00574 & GU256007 & Carolyn K Hurley, Washington DC, USA \\
\hline $\mathrm{CW}^{*} 0127$ & HN-35341-6 & GU128046 & Histogenetics, Ossining, USA \\
\hline $\mathrm{CW}^{*} 0132$ & $\begin{array}{l}\text { HN-14507-0, HN-81436-2, HN-39457-9, } \\
\text { HN-05381-9, HN-89122-6, HN-01820-8 }\end{array}$ & $\begin{array}{l}\text { GU017959, GQ161036, GQ180254, } \\
\text { GQ180418, GQ180420, GQ240482 }\end{array}$ & Histogenetics, Ossining, USA \\
\hline $\mathrm{CW}^{*} 020207$ & $\begin{array}{l}\text { HN-16328-8, HN-11201-0, HN-83016-5, } \\
\text { HN-56299-2, HN-75876-6 }\end{array}$ & $\begin{array}{l}\text { GU128039, GQ161064, GQ161067, } \\
\text { GQ161069, GQ180442 }\end{array}$ & Histogenetics, Ossining, USA \\
\hline $\mathrm{CW}^{*} 020208$ & $\mathrm{HN}-05717-7$ & GQ180244 & Histogenetics, Ossining, USA \\
\hline $\mathrm{CW}^{*} 021602$ & HN-58658-8 & GU017962 & Histogenetics, Ossining, USA \\
\hline $\mathrm{CW}^{*} 0230$ & $\mathrm{HN}-19588-2, \mathrm{HN}-09354-8$ & GQ161065, GQ994080 & Histogenetics, Ossining, USA \\
\hline $\mathrm{CW}^{*} 0231$ & $\mathrm{HN}-42278-2$ & GQ180394 & Histogenetics, Ossining, USA \\
\hline $\mathrm{CW}^{*} 030307$ & HN-18083-7 & GU128023 & Histogenetics, Ossining, USA \\
\hline $\mathrm{CW}^{*} 030308$ & $H N-53315-0$ & G0994082 & Histogenetics, Ossining, USA \\
\hline $\mathrm{CW}^{*} 0321$ & BY00570 & GU256003 & Carolyn K Hurley, Washington DC, USA \\
\hline $\mathrm{CW}^{*} 033802$ & BY00571 & GU256004 & Carolyn K Hurley, Washington DC, USA \\
\hline $\mathrm{CW}^{*} 0374$ & HN-36900-8 & GU128026 & Histogenetics, Ossining, USA \\
\hline $\mathrm{CW}^{*} 0379$ & $\mathrm{HN}-22447-9$ & G0994083 & Histogenetics, Ossining, USA \\
\hline $\mathrm{CW}^{*} 040108$ & HN-69805-2, HN-03781-6 & GU017945, GQ180269 & Histogenetics, Ossining, USA \\
\hline $\mathrm{CW}^{*} 040108$ & AKB-651323 & FN430727 & Rainer Blasczyk, Hannover, Germany \\
\hline $\mathrm{CW}^{*} 040110$ & $\begin{array}{l}\text { HN-15468-3, HN-11404-8, HN-80358-7, } \\
\text { HN-63809-2, HN-03817-1 }\end{array}$ & $\begin{array}{l}\text { GU128038, GQ161049, GQ180259, } \\
\text { GQ180270, GQ254388 }\end{array}$ & Histogenetics, Ossining, USA \\
\hline
\end{tabular}


Table 2 Continued

\begin{tabular}{|c|c|c|c|}
\hline Sequence & Cell identification & Accession number & Submitting author \\
\hline $\mathrm{CW}^{*} 040113$ & HN-68703-0, HN-17952-4 & GU017944, GU128022 & Histogenetics, Ossining, USA \\
\hline $\mathrm{CW}^{*} 040115$ & $\mathrm{HN}-73887-7$ & GQ994086 & Histogenetics, Ossining, USA \\
\hline $\mathrm{Cw}^{*} 0439$ & HN-80328-7 & GQ240486 & Histogenetics, Ossining, USA \\
\hline $\mathrm{CW}^{*} 0448$ & $H N-50961-3$ & GQ254395 & Histogenetics, Ossining, USA \\
\hline $\mathrm{CW}^{*} 0452$ & HN-25199-0 & GQ994087 & Histogenetics, Ossining, USA \\
\hline $\mathrm{CW}^{*} 050107$ & HN-28304-6 & GU017961 & Histogenetics, Ossining, USA \\
\hline $\mathrm{CW}^{*} 050108$ & HN-65756-1 & GU128049 & Histogenetics, Ossining, USA \\
\hline $\mathrm{CW}^{*} 0526$ & N-671307 & FN430728 & Rainer Blasczyk, Hannover, Germany \\
\hline $\mathrm{CW}^{*} 0526$ & $\mathrm{HN}-08336-1$ & GQ240509 & Histogenetics, Ossining, USA \\
\hline $\mathrm{CW}^{*} 0529$ & $H N-54907-5$ & GQ180263 & Histogenetics, Ossining, USA \\
\hline $\mathrm{CW}^{*} 0534$ & HN-27839-1 & GU017940 & Histogenetics, Ossining, USA \\
\hline $\mathrm{CW}^{*} 0623$ & HN-68335-1, HN-35876-3 & GU128024, GQ161071 & Histogenetics, Ossining, USA \\
\hline $\mathrm{CW}^{*} 0626$ & $\mathrm{HN}-74025-2, \mathrm{HN}-72178-4$ & GQ180265, GQ254381 & Histogenetics, Ossining, USA \\
\hline $\mathrm{CW}^{*} 0627$ & HN-28905-8 & GQ180431 & Histogenetics, Ossining, USA \\
\hline $\mathrm{CW}^{*} 070110$ & HN-62495-1 & GQ161041 & Histogenetics, Ossining, USA \\
\hline $\mathrm{CW}^{*} 070204$ & HN-66407-8 & GQ180390 & Histogenetics, Ossining, USA \\
\hline $\mathrm{CW}^{*} 070210$ & $\begin{array}{l}\text { HN-45647-3, HN-04512-1, HN-25864-1, } \\
\text { HN-20592-7 }\end{array}$ & $\begin{array}{l}\text { GU017943, GU128018, GQ161070, } \\
\text { G0994089 }\end{array}$ & Histogenetics, Ossining, USA \\
\hline $\mathrm{CW}^{*} 070211$ & HN-13689-5 & GQ180446 & Histogenetics, Ossining, USA \\
\hline $\mathrm{CW}^{*} 0768$ & $\begin{array}{l}\text { HN-53344-0, HN-28940-6, HN-76839-2, } \\
\text { HN-91790-5, HN-40383-4 }\end{array}$ & $\begin{array}{l}\text { GU017950, GQ180252, GQ240505, } \\
\text { G0994073, GQ994084 }\end{array}$ & Histogenetics, Ossining, USA \\
\hline $\mathrm{CW}^{*} 0773$ & HN-36623-6, HN-01145-2 & GU128037, GQ240515 & Histogenetics, Ossining, USA \\
\hline $\mathrm{CW}^{*} 0776$ & HN-38523-1 & GQ161045 & Histogenetics, Ossining, USA \\
\hline $\mathrm{Cw}^{*} 0785$ & $H N-24541-5$ & GQ161031 & Histogenetics, Ossining, USA \\
\hline $\mathrm{CW}^{*} 0791$ & HN-39440-4, HN-55690-4, HN-27500-6 & GQ161072, GQ180250, GQ240481 & Histogenetics, Ossining, USA \\
\hline $\mathrm{CW}^{*} 0828$ & HN-13242-5, HN-30318-9, HN-48864-2 & GU017957, GQ161061, GQ240491 & Histogenetics, Ossining, USA \\
\hline$C w^{*} 120308$ & $\mathrm{HN}-84987-5$ & GQ240483 & Histogenetics, Ossining, USA \\
\hline $\mathrm{CW}^{*} 1227$ & $\mathrm{HN}-74768-3$ & GQ149306 & Histogenetics, Ossining, USA \\
\hline $\mathrm{CW}^{*} 1414$ & HN-09762-5 & GQ254392 & Histogenetics, Ossining, USA \\
\hline$C W^{*} 1507$ & BY00572 & GU256005 & Carolyn K Hurley, Washington DC, USA \\
\hline $\mathrm{CW}^{*} 1523$ & $H N-30227-5$ & GQ240516 & Histogenetics, Ossining, USA \\
\hline$C W^{*} 160104$ & HN-N264212 & GQ994077 & Histogenetics, Ossining, USA \\
\hline$C W^{*} 1614$ & HN-65879-2 & GQ180444 & Histogenetics, Ossining, USA \\
\hline $\mathrm{CW}^{*} 1615$ & HN-10995-9, HN-25373-9 & GQ180434, GQ254384 & Histogenetics, Ossining, USA \\
\hline$C w^{*} 1616$ & $\mathrm{HN}-43823-8$ & GQ161050 & Histogenetics, Ossining, USA \\
\hline DRB1*133303 & P-658554 & FN430726 & Rainer Blasczyk, Hannover, Germany \\
\hline DPB1*1001 & 194229 & FN594947 & Klaus Witter, München, Germany \\
\hline DPB1*110101 & 171880 & FN598969 & Klaus Witter, München, Germany \\
\hline $\mathrm{DPB} 1 * 3001$ & 170324 & FN594948 & Klaus Witter, München, Germany \\
\hline
\end{tabular}

Table 3 Recently Published Sequences

\begin{tabular}{lc}
\hline Sequence & References \\
\hline$B^{*} 4088$ & $(1)$ \\
$B^{*} 5214$ & $(2)$ \\
$C W^{*} 040110$ & $(3)$ \\
$C W^{*} 0442$ & $(4)$ \\
$C W^{*} 0624$ & $(5)$ \\
$C W^{*} 0774$ & $(6)$ \\
$C W^{*} 0766$ & $(7)$ \\
$C W^{*} 0767$ & $(7)$ \\
$C W^{*} 0824$ & $(8)$ \\
$D R B 1 * 0337$ & $(9)$ \\
$D R B 1 * 1533$ & $(10)$ \\
DOB $1 * 060105$ & $(6)$ \\
\hline
\end{tabular}

have been assigned by the data-libraries and most sequences are already available, there is still the possibility that an author may not yet have allowed the sequence to be released; in such a case you will have to contact the submitting author directly. Additional information pertaining to new sequences is often included in the publications describing these alleles; a listing of recent publications that describe new HLA sequences is given in Table 3.

All new and confirmatory sequences should now be submitted directly to the WHO Nomenclature Committee for Factors of the HLA System via the IMGT/HLA Database using the sequence submission tool provided. The IMGT/HLA Database may be accessed via the World Wide Web at: www.ebi.ac.uk/imgt/hla 


\section{References}

1. Yan LX, Zhu FM, Zhang W, He J. HLA-B*4088, a new allele was identified by polymerase chain reaction sequence-based typing in a Chinese cord blood donor. Tissue Antigens 2010: 75: 79-80.

2. Chen MJ, Chu CC, Shyr MH, Lin CL, Lin PY, Yang KL. A novel HLA-B allele, B*5214, detected in a Taiwanese volunteer bone marrow donor using a sequence-based typing method. Int J Immunogenet 2010: 37: 39-41.

3. Thude H, Michael M, Loth M, Schorner U, Barz D. Identification of the novel allele, $\mathrm{Cw}^{*} 040110$ in a German patient. Tissue Antigens 2010: 75: 80-2.

4. Balas A, Garcia-Sanchez F, Vicario JL. The new HLA-Cw*0442 allele possibily generated by a recombination event involving $\mathrm{CW}^{*} 04010101$ and $\mathrm{Cw}^{*} 1801$. Tissue Antigens 2010: 75: 82-3.

5. Deng ZH, Wang DM, Gao SQ, Xu YP. Characterization of the novel HLA-Cw*0624 allele identified by sequence-based typing. Tissue Antigens 2010: 75: 83-4.
6. Endres RO, Redman H, Marcus N. Identification of the HLA-Cw*0774 and DQB1*060105 alleles. Tissue Antigens 2010: 75: 84-6.

7. Deng Z, Xu Y, Wang D, Jin S. Description of two novel alleles HLA-Cw*0766 and $\mathrm{Cw}^{*} 0767$ identified in a Chinese Han individual by sequence-based typing. Hum Immunol 2010: 71: $93-5$.

8. Wang DM, Zou HY, Li Z. Identification of a novel HLA-Cw*08 variant allele, $\mathrm{Cw}^{*} 0824$. Tissue Antigens 2010: 75: $86-7$.

9. Chen MJ, Chu CC, Shyr MH, Lin CL, Lin PY, Yang KL. Detection of a rare Caucasoid HLA-DRB $1 * 0337$ in a Taiwanese bone marrow donor using sequence-based typing method. Int J Immunogenet 2010: 37: $1-3$.

10. Parkner A, Mosebach M, Halm-Heinrich I, Heim M. Identification of a novel HLA-DRB1*15 allele, DRB1*1533, in a recently registered stem cell donor. Tissue Antigens 2010: 75: $88-9$. 